\title{
Marcadores de demencia en la enfermedad de Parkinson
}

\author{
María José Martí Domènech \\ Coordinadora de la Unidad de Parkinson y Trastornos del Movimiento. Servicio de Neurología. \\ Institut Clínic de Neurociències (ICN). Centro de Investigación en Red de Enfermedades Neurodegenerativas (CIBERNED). \\ Hospital Clínic de Barcelona.
}

La demencia es un síntoma no motor que van a presentar una gran parte de los pacientes con la enfermedad de Parkinson (EP) a lo largo del curso de la enfermedad, aunque tanto la rapidez del declive cognitivo como el inicio de demencia pueden ser muy variables. Los déficits cognitivos tienen importantes consecuencias tanto para los pacientes como para sus familiares ${ }^{1}$, y representan una importante carga social y sanitaria. Aunque se puede lograr una mejoría sintomática con fármacos como los inhibidores de la acetilcolinesterasa ${ }^{2}$, hasta el momento carecemos de terapias capaces de prevenir su desarrollo o enlentecer su progresión. La identificación de marcadores de riesgo de demencia, ya sean biológicos, clínicos o de neuroimagen, podría facilitar el desarrollo de este tipo de terapias.

En este número de Alzheimer: Realidades e investigación en demencia, Ibarretxe y Junque ${ }^{3}$ presentan los resultados de un estudio longitudinal, cuyo objetivo es investigar si la presencia de alucinaciones visuales (AV) y la pérdida de volumen de la sustancia gris hipocampal es un factor de riesgo de demencia en la EP. Previamente las autoras habían observado que los pacientes con EP y demencia presentaban una afectación de cabeza y cola de la sustancia gris hipocampal, con relativa preservación del cuerpo, y que esta alteración estaba limitada a la cabeza en los pacientes con EP no dementes pero con AV, lo que podía indicar que la neurodegeneración de esta estructura empezaba en la cabeza y posteriormente afectaba la parte

Correspondencia: M. J. Martí Domènech.

E-mail:mjmarti@clinic.ub.es posterior ${ }^{4}$. Después de un seguimiento medio de más de 2 años, el 73\% de los pacientes con AV cumplían criterios de demencia comparado con ninguno en el grupo de pacientes sin AV. También en este grupo de pacientes se observaba una atrofia difusa hipocampal, con una extensión antero-posterior, comparado con el grupo control. Ningún paciente sin AV cumplía criterios de demencia y sólo 3 pacientes, con mayor déficit en memoria, mostraron atrofia del hipocampo, de nuevo comparado con el grupo control.

Este estudio, y otros previos de los mismos investigadores y de otros ${ }^{5,6}$, son importantes porque ponen de relieve la afectación de estructuras corticales, sobre todo del lóbulo temporal medial pero también del cíngulo y la corteza parieto-occipital, en la demencia asociada a EP. Estos hallazgos, junto a estudios patológi$\cos ^{7-9}$ en los que se ha observado una asociación entre demencia y lesiones de tipo Alzheimer o agregados de alfa-sinucleína, en regiones límbicas y corticales, han modificado el concepto de que la demencia en la EP era de tipo subcortical debido a la pérdida neuronal en estructuras monoaminérgicas y colinérgicas.

En este estudio sólo los pacientes que presentaban $\mathrm{AV}$ evolucionaron a una demencia y únicamente en ellos se observó la progresión de la atrofia hipocampal en el seguimiento. Aunque las AV se han invocado como factores de riesgo, también lo han sido la presencia de alteraciones cognitivas en la memoria y el lenguaje, la edad del paciente en el momento del estudio, o el tipo y la gravedad del parkinsonismo ${ }^{10}$. En un elevado porcentaje, los pacientes de este estudio alcanzaron la demencia en un tiempo relativamente corto, lo que podría estar indicando que ya presentaban un claro déficit cognitivo basal, como se desprende de las 
puntuaciones en los test neuropsicológicos. Estos datos sugieren que no sólo las alucinaciones, sino también el patrón de deterioro cognitivo serían factores de riesgo en este grupo de pacientes, y que su correlato anatómico subyacería en el hipocampo y en otras posibles estructuras relacionadas con las alteraciones de la percepción.

Una limitación del estudio es el pequeño número de pacientes estudiados, limitación a veces imposible de superar dado el carácter progresivo e invalidante de la enfermedad, pero que dificulta la generalización de los hallazgos. Además, ni todos los pacientes con demencia presentaban atrofia hipocampal, ni todos los que presentaban atrofia tenían demencia, lo que podría interpretarse como que la demencia en la EP puede tener una causa heterogénea o que los cambios estructurales aparecen antes que la clínica se manifieste; en este sentido sí podría considerarse un buen marcador de demencia. Sin embargo, la atrofia hipocampal observada no fue el resultado de comparar los pacientes parkinsonianos entre sí, sino cada grupo por separado con los sujetos controles. Probablemente no existían diferencias significativas en la medición hipocampal entre ambos grupos de pacientes parkinsonianos, por lo que su utilidad como marcador de demencia en el ámbito clínico no sería aplicable. Posiblemente, marcadores moleculares que sean incluso más precoces que los cambios estructurales, como biomarcadores en el líquido cefalorraquídeo o captados mediante nuevas técnicas de imagen PET (tomografía por emisión de positrones), puedan representar un paso más en la detección precoz de la demencia o, aún más importante, en la identificación de individuos en riesgo de desarrollarla ${ }^{11}$. A pesar de estas limitaciones metodológicas, los resultados del estudio ponen de manifiesto la necesidad de profundizar en la neuroimagen como biomarcador de demencia, ya sea sola o combinada con otros posibles marcadores de riesgo.

\section{Bibliografía}

1. Aarsland D, Brønnick K, Ehrt U, De Deyn PP, Tekin S, Emre M, et al. Neuropsychiatric symptoms in patients with Parkinson's disease and dementia: frequency, profile and associated care giver stress. J Neurol Neurosurg Psychiatry. 2007;78:36-42.

2. Emre M, Aarsland D, Albanese A, Byrne EJ, Deuschl G, De Deyn PP, et al. Rivastigmine for dementia associated with Parkinson's disease. N Engl J Med. 2004:351:2509-18.

3. Ibarretxe-Bilbao N, Junque C. ¿Es la atrofia del hipocampo en la enfermedad de Parkinson un predictor de demencia? Alzheimer. Real Invest Demenc. 2011;

4. Ibarretxe-Bilbao N, Ramírez-Ruiz B, Tolosa E, Martí MJ, Valldeoriola F, Bargalló N, et al. Hippocampal head atrophy predominance in Parkinson's disease with hallucinations and with dementia. J Neurol. 2008;255:1324-31.

5. Junqué C, Ramírez-Ruiz B, Tolosa E, Summerfield C, Martí MJ, Pastor P, et al. Amygdalar and hippocampal MRI volumetric reductions in Parkinson's disease with dementia. Mov Disord. 2005;20:540-4.

6. Bouchard TP, Malykhin N, Martin WR, Hanstock CC, Emery DJ, Fisher NJ, et al. Age and dementia-associated atrophy predominates in the hippocampal head and amygdala in Parkinson's disease. Neurobiol Aging. 2008;29: 1027-39.

7. SantaCruz K, Pahwa R, Lyons K, Troster A, Handler M, Koller W, et al. Lewy body, neurofibrillary tangle and senile plaque pathology in Parkinson's disease patients with and without dementia. Neurology. 1999;52(suppl 2): A476-7.

8. Jellinger KA, Seppi K, Wenning GK, Poewe W. Impact of coexistent Alzheimer pathology on the natural history of Parkinson's disease. J Neural Transm. 2002;109:329:39.

9. Braak H, Rüb U, Jansen Steur ENH, Del Tredici K, De Vos RA. Cognitive status correlates with neuropathologic stage in Parkinson disease. 2005;64:1404-10.

10. Caballol N, Martí MJ, Tolosa E. Cognitive dysfunction and dementia in Parkinson disease. Mov Disord. 2007; Suppl 17:S358-66.

11. Siderowf A, Xie SX, Hurtig H, Weintraub D, Duda J, Chen-Plotkin A, et al. CSF amyloid \{beta\} 1-42 predicts cognitive decline in Parkinson disease. Neurology. 2010; 75(12):1055-61 